Gefässchirurgie 2010 · 15:4-4

DOI 10.1007/s00772-009-0778-x

๑) Springer-Verlag 2010

\author{
E. Sebastian Debus \\ Klinik und Poliklinik für Gefäßmedizin, Universitäres Herzzentrum GmbH, \\ Universitätsklinikum, Hamburg-Eppendorf
}

\title{
Was gibt es Neues in 2010?
}

\section{Mehr Hefte, neue Rubriken und ein attraktiver Preis}

Liebe Leserinnen und Leser,

die Gefässchirurgie - Zeitschrift für vaskuläre und endovaskuläre Medizin hat sich innerhalb der nahezu unüberschaubaren Vielzahl medizinischer Fachzeitschriften einen festen Platz gesichert und erfreut sich zunehmender Beliebtheit, was sich durch einen kontinuierlich steigenden Leserkreis - auch außerhalb unserer Fachkollegen - zeigt.

\section{Listung im Science Citation Index}

Die Listung im Science Citation Index war ein wichtiger Meilenstein auf diesem Weg; in Kürze erwarten wir die erste offizielle Impactbewertung unserer Beiträge, die dann von 2007 an gelten wird.

\section{Jetzt 8 Ausgaben pro Jahr}

In diesem Jahr erwartet unseren Leserkreis eine Reihe von Weiterentwicklungen der Zeitschrift. Auf der vergangenen Herausgebersitzung im Oktober 2009 in München wurde beschlossen, der Zunahme unserer Einsendungen durch ein Heraufsetzen der Erscheinungsweise ab 2010 von 6 Hefte auf 8 Hefte pro Jahr Rechnung zu tragen.

\section{Springer Award Gefäßmedizin}

Dazu kommt ein attraktiver Wissenschaftspreis, der Springer Award, den der gleichnamige Verlag auslobt. Er wird erstmals anlässlich der Dreiländertagung vom 7.-11. September 2010 in Berlin vergeben. Es handelt sich um ein Reisestipendium in Höhe von $2.500 €$ für den besten experimentellen Forschungsbeitrag aus der Gefäßmedizin. Er ist geknüpft an die Einsendung eines Manuskriptes, das spätestens drei Monate vor der Jahrestagung bei der Schriftleitung der Gefässchirurgie eingegangen sein muß, und an einen Vortrag auf der Springer Award Preisträgersitzung während des Kongresses. Eine unabhängige Jury aus dem Herausgeberboard der Zeitschrift prämiert den besten Beitrag; die besten vier Manuskripte werden in einem eigenen Schwerpunktheft (im Anschluss an die Kongressausgabe) veröffentlicht. Die weiteren Details hierzu sind auf der Homepage der DGG einzusehen.

\section{Rubrikenspektrum erweitert}

Des Weiteren werden wir zwei neue Rubriken in das Portfolio unserer Zeitschrift aufnehmen: Wir werden jeweils eine Übersichtsarbeit (Review) zu wichtigen Themen bringen, die im Heft vor den Leitthemenbeiträgen angesiedelt ist. Dieser Schritt ist erforderlich geworden, weil die Evidenzlage in unserem Fach stetig steigt und neue Entwicklungen eine regelmäßige wissenschaftliche Neubewertung nach Datenlage der Literatur erfordert. Die Rubrik Fragen aus der Praxis diskutiert Entscheidungsbäume auf Basis aktueller Daten aus der Literatur anhand eines klinischen Falles und kommt so zu evidenzbasierten Lösungsansätzen. Die Rubrik wird federführend von Herrn Kollegen Dr. H. Böhner geleitet, ein Engagement, das Regelmäßigkeit und Nachhaltigkeit erfordert und wofür wir ihm sehr dankbar sind!

Wir sind sicher, dass diese Neuerungen weiter zu der Entwicklung der Gefässchirurgie beitragen werden!

Ihr

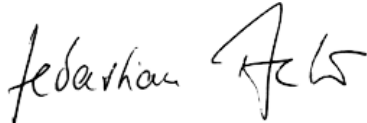

E. Sebastian Debus

\section{Korrespondenzadresse}

Prof. Dr. med. E. Sebastian Debus

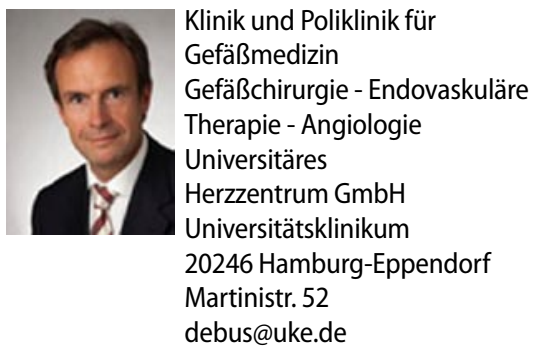

